\title{
Four new subdwarf B pulsators ${ }^{\star, \star \star, \star \star \star}$
}

\author{
R. Østensen ${ }^{1,4}$, U. Heber ${ }^{2}$, R. Silvotti ${ }^{3}$, J.-E. Solheim ${ }^{4}$, S. Dreizler ${ }^{5}$, and H. Edelmann ${ }^{2}$ \\ 1 Isaac Newton Group of Telescopes, 37800 Santa Cruz de La Palma, Canary Islands, Spain \\ 2 Dr. Remeis-Sternwarte, Astronomisches Institut der Universität Erlangen-Nürnberg, 96049 Bamberg, Germany \\ e-mail: ai03@sternwarte.uni-erlangen.de; edelmann@sternwarte.uni-erlangen.de \\ 3 Osservatorio Astronomico di Capodimonte, via Moiariello 16, 80131 Napoli, Italy \\ e-mail: silvotti@na.astro.it \\ ${ }^{4}$ Institutt for Fysikk, Universitetet i Troms $\varnothing, 9037$ Troms $\varnothing$, Norway \\ e-mail: janerik@phys .uit.no \\ 5 Institut für Astronomie und Astrophysik, Waldhäuser Straße 64, 72076 Tübingen, Germany \\ e-mail: dreizler@astro.uni-tuebingen.de
}

Received 13 June 2001 / Accepted 28 August 2001

\begin{abstract}
We report the detection of short period oscillations in the sdB stars HS 0039+4302, HS 0444+0408, HS $1824+5745$ and HS $2151+0857$ from time-series photometry made at the Nordic Optical Telescope (NOT) of a sample of 55 candidates. Hence these four hot subdwarfs are new members of the EC 14026 class of pulsating sdB stars. HS 0039+4302 is a multi-mode pulsator with at least four distinct periods in the range between 182 and $234 \mathrm{~s}$, and amplitudes up to 8 mma. HS 0444+0408 shows one dominant pulsation at $137 \mathrm{~s}(A \sim 12 \mathrm{mma})$ and a second weaker pulsation at $170 \mathrm{~s}(A \sim 3 \mathrm{mma})$. For HS $1824+5745$ we find a single period of $139 \mathrm{~s}$ with an amplitude of about 5 mma. HS $2151+0857$ shows four periods in the range 129-151 s with amplitudes between 2 and 5 mma. Our NLTE model atmosphere analysis of the time-averaged optical spectra place all stars well within the theoretical sdBV instability strip.
\end{abstract}

Key words. subdwarfs - stars: oscillations - stars: individual: HS 0039+4302, HS 0444+0408, HS 1824+5745, HS $2151+0857$

\section{Introduction}

Subluminous B stars (sdB) dominate the populations of faint blue stars of our own Galaxy and are found in both the old disk (field sdBs) and in halo populations as blue tails to the horizontal branches of globular clusters (Ferraro et al. 1997). Observations of elliptical galaxies with the Ultraviolet Imaging Telescope (Brown et al. 1997) and the HST (Brown et al. 2000) have shown that these

\footnotetext{
Send offprint requests to: R. Østensen,

e-mail: roy@phys .uit.no

* Based on observations obtained at the Nordic Optical Telescope, operated on the island of La Palma jointly by Denmark, Finland, Iceland, Norway, and Sweden, in the Spanish Observatorio del Roque de los Muchachos of the Instituto de Astrofisica de Canarias.

$\star \star$ Based on observations collected at the German-Spanish Astronomical Center, Calar Alto, operated by the Max-PlankInstitute für Astronomie Heidelberg jointly with the Spanish National Commission for Astronomy.

$\star \star \star$ Based on observations collected at the European Southern Observatory, Chile (ESO No.66.D-0031).
}

stars are sufficiently common to be the dominant source for the "UV upturn phenomenon" observed in elliptical galaxies and galaxy bulges (see also Greggio \& Renzini 1999). However, important questions remain over the exact evolutionary paths and the appropriate timescales.

It is now generally accepted that the sdB stars can be identified with models for extreme Horizontal Branch (EHB) stars burning He in their core, but with a very tiny ( $<1 \%$ by mass) inert hydrogen envelope (Heber 1986 ; Saffer et al. 1994). An EHB star bears great resemblance to a helium main-sequence star of half a solar mass and its further evolution should proceed similarly (i.e. directly to the white dwarf graveyard), as confirmed by recent calculations (Dorman et al. 1993). Therefore sdB stars are certainly important as the immediate progenitors of low mass white dwarfs.

How they evolve to the EHB configuration is controversial. The problem is how the mass loss mechanism in the progenitor manages to remove all but a tiny fraction of the hydrogen envelope at precisely the same time as the He core has attained the mass $\left(\sim 0.5 M_{\odot}\right)$ required 

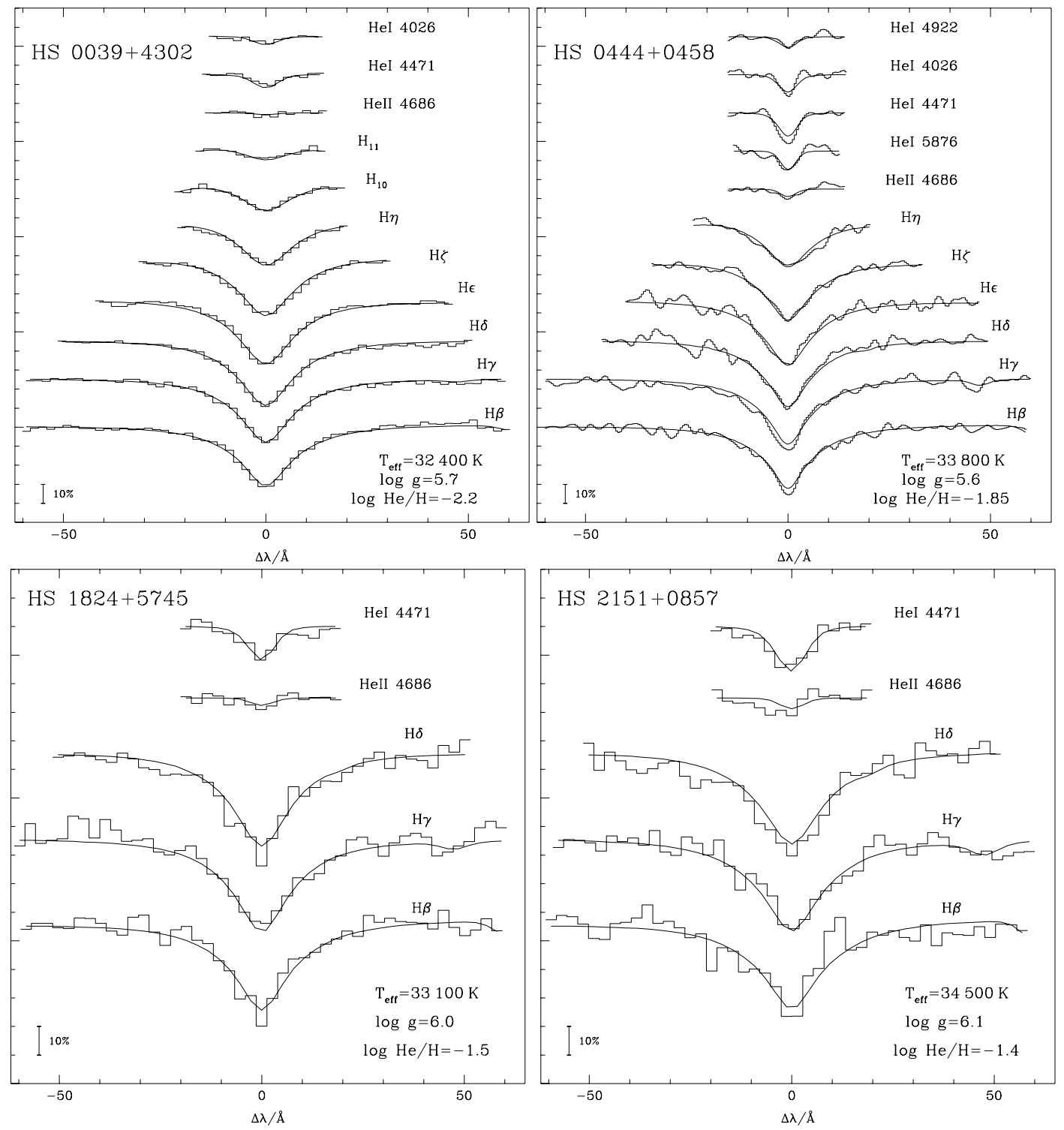

Fig. 1. Model fits to the Balmer and He line profiles in our time-averaged optical spectra of the four programme stars using NLTE model atmospheres (see text).

for the He flash. Considerable evidence is accumulating that many sdB stars reside in close binaries (Maxted et al. 2001) and therefore mass transfer in close binary evolution is important.

The discovery of multimode pulsators among the sdB stars has opened a new attractive possibility of probing their interiors using seismological methods. The properties of sdB pulsators ( $\mathrm{sdB}$ Variables $=$ sdBVs or EC 14026 stars, Kilkenny et al. 1997), are characterised by relatively short pulsation periods ranging between $\sim 1$ and $10 \mathrm{~min}$ and low pulsation amplitudes. PG $1605+072$ has the highest detected amplitude; the strongest observed peak has been found at 64 milli modulation amplitudes (mma), and more than 50 oscillation frequencies have been detected (Koen et al. 1998; Kilkenny et al. 1999). Most sdBVs have been found with a main peak pulsation amplitude of around $10 \mathrm{mma}$, while the lowest level pulsator discovered up to now is PG 1618+563B (Silvotti et al. 2000), with a main peak at only $2 \mathrm{mma}$.
The pulsations of the sdB stars are driven by an opacity bump associated with iron ionization (Charpinet et al. 1996, 1997). Both radial and nonradial modes are expected to have about the same range of frequencies, corresponding to what is observed (see also Kawaler 1999). The instability is predicted to occur in the temperature range between about $29000 \mathrm{~K}$ and $37000 \mathrm{~K}$. However, most stars in this temperature range do not vary. Our ongoing survey indicates that only one tenth of the sdB stars in this temperature interval is pulsating (Østensen 2000).

Today 19 sdBVs are known in the literature. 13 are summarised in the review by O'Donoghue et al. (1999), and six have recently been published (Piccioni et al. 2000; Billères et al. 2000; Silvotti et al. 2000; Østensen et al. 2001; Ulla et al. 2001). In this paper we present spectroscopy and photometry on another four, increasing the number of class members to 23 .

The four new pulsators reported in this paper have been identified based on a list of candidates drawn from 
Table 1. Details of spectroscopic observations. The last coloumn gives the size and identifier of the telescope used, with the spectrographs reciprocal dispersion in parentheses. (See text for details.) The $B$-band magnitudes given here have been estimated from the Schmidt plates.

\begin{tabular}{|c|c|c|c|c|c|c|}
\hline Object & $\begin{array}{c}B \\
\mathrm{mag}\end{array}$ & Date & $\begin{array}{c}\operatorname{Exp} \\
\mathrm{s}\end{array}$ & $\begin{array}{l}\lambda \text { range } \\
\AA\end{array}$ & $\begin{array}{l}\text { Dispersion } \\
\AA / \mathrm{mm}\end{array}$ & Observatory $^{\mathrm{a}}$ \\
\hline HS 0039+4302 & 15.1 & 3.10 .98 & 3000 & $4010-6720$ & 100 & $\mathrm{CA}$ \\
\hline \multirow[t]{3}{*}{ HS $0444+0458$} & 15.2 & 5.9 .93 & 2392 & $4010-6720$ & 120 & $\mathrm{CA}$ \\
\hline & & 15.10 .00 & 2400 & $3900-6930^{\mathrm{b}}$ & 110 & DK \\
\hline & & 16.10 .00 & 2400 & $3390-5620^{\mathrm{b}}$ & 110 & DK \\
\hline HS $1824+5745$ & 15.6 & 5.9 .93 & 4000 & $4010-6720$ & 120 & CA \\
\hline HS 2151+0857 & 16.5 & 5.9 .93 & 4000 & $4010-6720$ & 120 & $\mathrm{CA}$ \\
\hline
\end{tabular}

a CA: Calar Alto $2.2 \mathrm{~m}$, Spain, DK: Danish $1.5 \mathrm{~m}$, Chile.

b Not flux calibrated.

Table 2. Results of the spectroscopic analysis.

\begin{tabular}{llllll}
\hline Object & $T_{\text {eff }}$ & $\log g$ & $\log \left(\frac{n(\mathrm{He})}{n(\mathrm{H})}\right)$ & $\begin{array}{l}M(\mathrm{~V}) \\
\mathrm{mag}\end{array}$ & $\begin{array}{l}\text { Distance } \\
\mathrm{kpc}\end{array}$ \\
\hline $\mathrm{K}$ & & & & \\
\hline HS 0039+4302 & $32400 \pm 700$ & $5.70 \pm 0.10$ & $-2.20 \pm 0.20$ & 4.2 & 1.5 \\
HS 0444+0458 & $33800 \pm 1000$ & $5.60 \pm 0.15$ & $-1.85 \pm 0.20$ & 3.9 & 1.8 \\
HS 1824+5745 & $33100 \pm 1700$ & $6.0 \pm 0.25$ & $-1.52 \pm 0.20$ & 4.9 & 1.4 \\
HS 2151+0857 & $34500 \pm 1300$ & $6.1 \pm 0.25$ & $-1.37 \pm 0.20$ & 5.1 & 1.9 \\
\hline
\end{tabular}

Table 3. Detected pulsation frequencies and amplitudes.

\begin{tabular}{|c|c|c|c|c|c|c|c|c|c|}
\hline Object & $\begin{array}{l}\text { Date } \\
(2000)\end{array}$ & $F_{1}$ & $\begin{array}{l}F_{2} \\
\quad(\mathrm{ml}\end{array}$ & $\begin{array}{c}F_{3} \\
\mathrm{Iz})\end{array}$ & $F_{4}$ & $A_{1}$ & $\begin{array}{l}A_{2} \\
(\mathrm{mn} \\
\end{array}$ & $\begin{array}{l}A_{3} \\
\text { a) }\end{array}$ & $A_{4}$ \\
\hline \multirow[t]{4}{*}{ HS $0039+4302$} & Oct. $4-5$ & 5.17 & & 4.28 & & 10.3 & & 2.6 & \\
\hline & Oct. 5-6 & 5.14 & 5.50 & 4.27 & & 8.1 & 6.8 & 2.7 & \\
\hline & Oct. 7 & 5.13 & 5.46 & 4.27 & 5.29 & 8.0 & 5.4 & 2.6 & 3.0 \\
\hline & Combined & 5.14 & 5.48 & 4.27 & 5.21 & 8.0 & 5.4 & 2.6 & 1.9 \\
\hline \multirow[t]{4}{*}{ HS $0444+0458$} & Oct. 6 & 7.31 & 5.91 & & & 11.9 & 2.6 & & \\
\hline & Oct. 7 & 7.32 & 5.87 & & & 11.5 & 2.6 & & \\
\hline & Oct. 8 & 7.31 & 5.90 & & & 11.1 & 2.4 & & \\
\hline & Combined & 7.31 & 5.86 & & & 12.1 & 2.5 & & \\
\hline \multirow{4}{*}{ HS $1824+5745$} & Jul. 9 & 7.27 & & & & 2.6 & & & \\
\hline & Jul. 10 & 7.21 & & & & 4.5 & & & \\
\hline & Jul. 11 & 7.19 & & & & 4.8 & & & \\
\hline & Combined & 7.21 & & & & 4.0 & & & \\
\hline \multirow[t]{4}{*}{ HS $2151+0857$} & Jul. 9 & 7.40 & 7.74 & 6.59 & 7.01 & 4.8 & 2.1 & 2.8 & 2.5 \\
\hline & Jul. 10 & 7.39 & 7.80 & 6.58 & 6.88 & 4.8 & 2.6 & 3.1 & 3.1 \\
\hline & Jul. 11 & 7.42 & 7.73 & 6.62 & 6.85 & 4.8 & 4.6 & 3.6 & 2.5 \\
\hline & Combined & 7.43 & 7.72 & 6.59 & 6.86 & 5.0 & 3.3 & 3.3 & 2.1 \\
\hline
\end{tabular}

the Hamburg Schmidt survey (Hagen et al. 1995). Followup spectroscopy (Heber et al. 1999; Edelmann et al. 2001) revealed that their effective temperatures lie in the domain predicted for the pulsational instability. 13 objects were monitored with continuous photoelectric photometry with the Nordic Optical Telescope (NOT) in July 1999 and one pulsator, PG 1618+563B, was discovered (Silvotti et al. 2000). In October 1999, in a second run at the NOT, we used a CCD camera with our own controller software (Østensen \& Solheim 2000) to observe
31 additional candidates, of which three were identified as pulsators: HS 0815+4243, HS 2149+0847 and HS 2201+2610 (Østensen et al. 2001). The third run was completed in July 2000, when 24 candidates were observed during four nights, resulting in two of the discoveries presented in this paper. During the fourth run, three months later, 31 new candidates were observed and another 8 reobserved, revealing another two new pulsators. A more detailed description of the technique used, and upper limits to the pulsation amplitudes of the sdBs for which we 
do not see any pulsations above the $3 \sigma$ detection limit, will be reported in a subsequent paper when our search programme will be completed.

\section{The new sdBV stars}

Short period oscillations in the sdB stars HS $0039+4302$, HS $0444+0408$, HS $1824+5745$ and HS $2151+0857$ have been discovered by time-series photometry at the NOT. We have searced the SIMBAD catalouge for any previous observations and classifications of these stars, and for HS $0444+0408$ and HS $1824+5745$ the search comes out empty. For the two other stars, the results are as follows.

\subsection{HS $0039+4302$}

The SIMBAD catalogue lists a few registrations that match the coordinates of HS 0039+4302. Both the Balloon far-ultraviolet survey (Bixler et al. 1991) and the first Byurakan spectral sky survey (Abrahamian et al. 1990) list this object as a UV-excess object, with catalogue numbers of Balloon 84041013 and FBS 0039+430 = FBS B 210, respectively. No further references have been found in the literature.

\subsection{HS $2151+0857$}

A search in the SIMBAD catalogue around the coordinates of HS $2151+0857$ reveals that the sdB star PG 2151+089 should be less than two arcminutes away. Our images reveal no other blue stars in this region, so we conclude that the coordinates of PG 2151+089 are wrong and that this is actually the same star. The PG catalogue $B$-band magnitude of 16.31 (Green et al. 1986) is also in accordance with the value $B=16.5$, estimated from the Schmidt plates. According to SIMBAD, PG 2151+089 is listed in the Catalogue of spectroscopically identified hot subdwarf stars (Kilkenny et al. 1988).

\section{Spectroscopy}

Medium resolution (4-6 A) spectra were obtained in 1993 at the Calar Alto $2.2 \mathrm{~m}$ telescope equipped with the B\&C Cassegrain spectrograph, and in 1998 at the same telescope but using the CAFOS spectrograph. Details of the observations are listed in Table 1. Besides the Balmer lines of hydrogen, He I lines and He II $4686 \AA$ are detected in all stars. The exposure times are much longer than their oscillation periods (see Table 3) and therefore the spectra are time-averaged. Due to the poor quality of the spectrum of HS 0444+0458 we re-observed the star with the DFOSC at the Danish $1.5 \mathrm{~m}$ telescope at ESO. Spectra with two grisms (\#6 and \#7) were taken to cover the whole Balmer series.

The available spectra of HS $1824+5745$ and HS $2151+0857$ are of lower quality than those of the other two stars, and cover a smaller wavelength range,

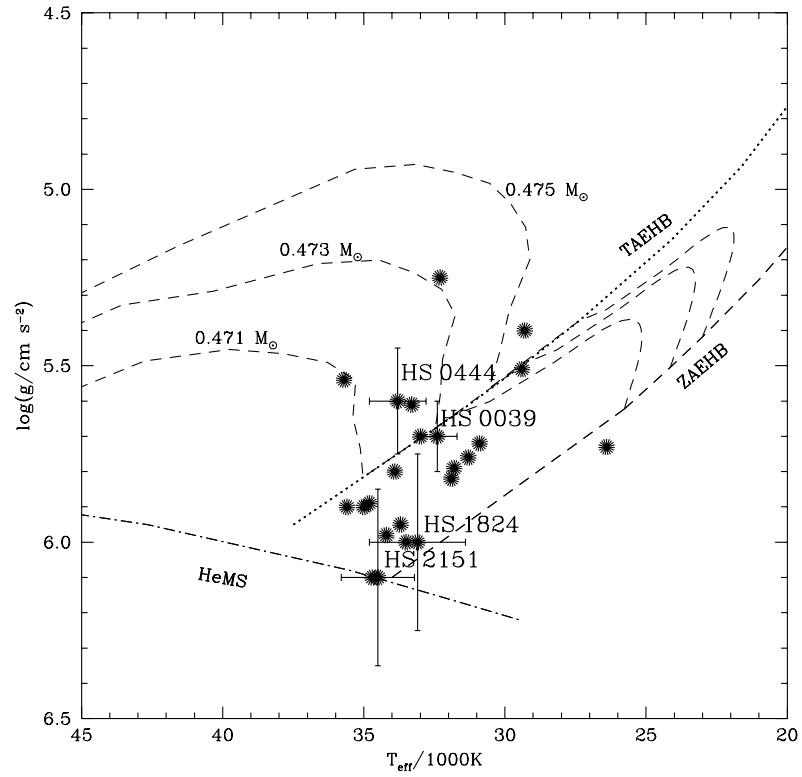

Fig. 2. Comparison of the four newly discovered sdBVs to previously known ones in the $\left(T_{\text {eff }}, \log g\right)$ plane. The position of the Zero Age Extreme Horizontal Branch (ZAEHB), the Terminal Age Extreme Horizontal Branch (TAEHB), the Helium Main Sequence (HeMS) and evolutionary tracks for EHB stars (Dorman et al. 1993) are also shown.

which allows to use only $\mathrm{H} \beta$ to $\mathrm{H} \delta$ for the spectral analysis.

A grid of synthetic spectra derived from $\mathrm{H}-\mathrm{He}$ line blanketed NLTE model atmospheres (Napiwotzki 1997) was matched to the data to simultaneously determine effective temperature, gravity and He abundance (Heber et al. 2000). The line profile fits are displayed in Fig. 1 and the resulting atmospheric parameters are listed in Table 2. Statistical fitting errors are low for HS $0039+4302$ and HS 0444+0458. However, systematic errors, which can only be estimated quantitatively from repeated observations, contribute significantly to the error budget. The error estimates in Table 2 are based on our previous experience (see Østensen et al. 2001). Adopting the canonical mass of $0.5 M_{\odot}$ for the sdB, we can estimate their absolute visual magnitudes and distances (as listed in Table 2).

\subsection{Discussion}

The determined temperatures, gravities and helium abundances are typical for pulsating sdB stars, as it can be seen when comparing with known sdBVs (Fig. 2).

HS $0039+4302$ is located right in the centre of the $\operatorname{sdBV}\left(T_{\text {eff }}, \log g\right)$ distribution at a position close to PG 1336-018 (Kilkenny et al. 1998).

The position of HS $0444+0408$ is similar to that of KPD 1930+2752 (Billères et al. 2000) to within the error bars.

HS $1824+5745$ has effective temperature and gravity close to that of EC 10228-0905 (Stobie et al. 1997). 


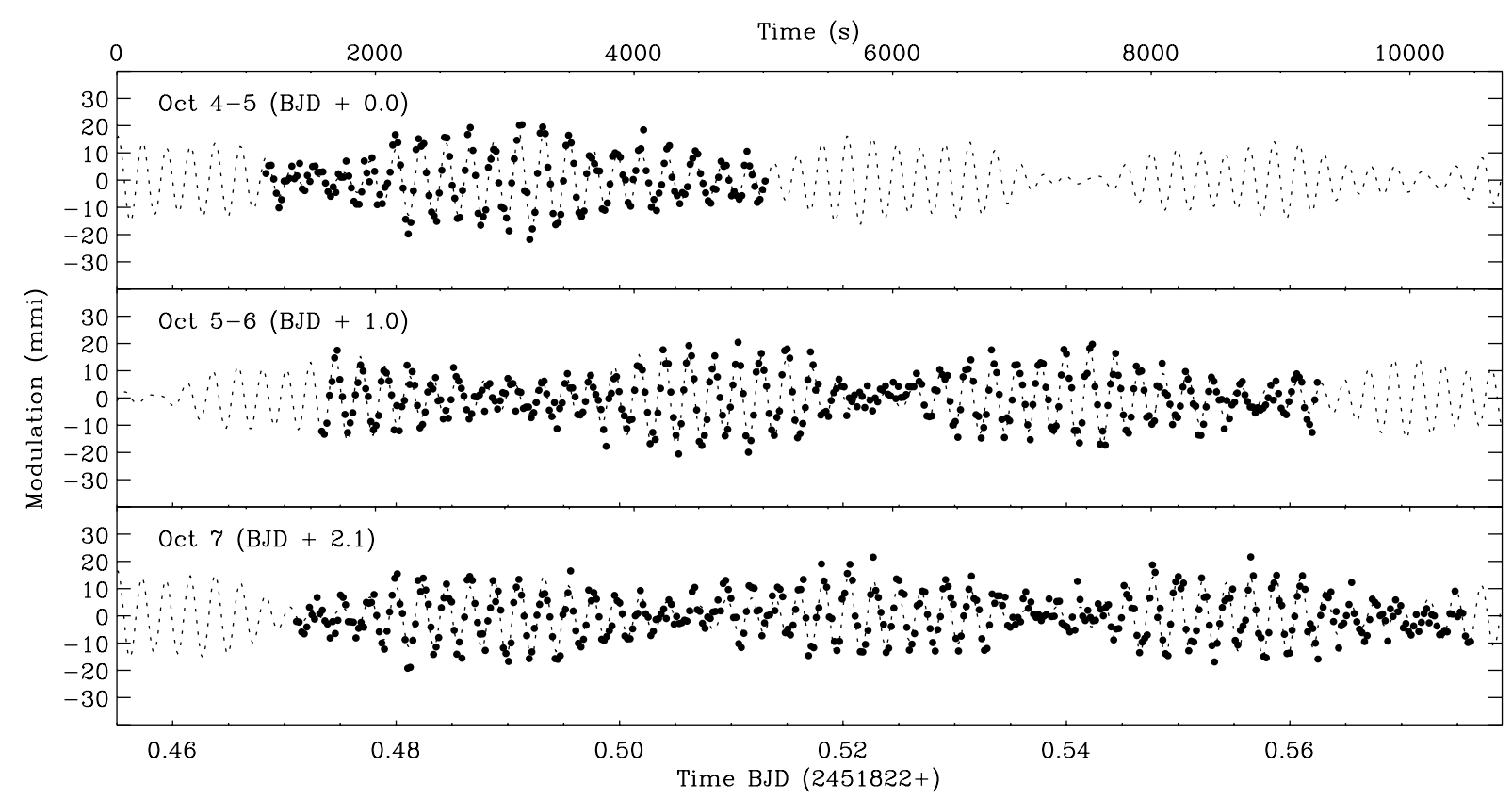

Fig. 3. Observed and synthetic light curves for HS 0039+4302. The scale on the $x$-axis above the plot shows time in seconds with an arbitrary zero point, and the scale on the bottom shows absolute time of observation in BJD when the numbers in parentheses are added.

HS 2151+0857 has similar effective temperature and gravity as the class prototype EC 14026-2647 (Kilkenny et al. 1997), and forms the high gravity edge of the known sdB stars in the $\left(T_{\text {eff }}, \log g\right)$ diagram (see Fig. 2$)$.

No indications of any binary companion stars are found in the spectra of any of these four sdB stars. Neither absorption lines such as the $\mathrm{Mg}$ I triplet, the $G$ band or $\mathrm{Ca} I$ $4226 \AA$ can be detected nor is there any flux excess in the red part of the spectra which could be caused by late type companions. Any companion therefore must be considerably fainter than the sdB stars whose absolute visual magnitudes are in the range $M_{V}=4-5 \mathrm{mag}$ (see Table 2). The spectra are not suitable for radial velocity measurements and therefore we cannot test whether the star that we have multiple spectra of, HS $0444+0408$, is a radial velocity variable or not.

\section{Time-series photometry}

The observations were done at the Nordic Optical Telescope (NOT) with the Andalucia Faint Object Spectrograph and Camera (ALFOSC), equipped with a Loral, Lesser thinned, $2048 \times 2048$ CCD chip, and modified with our own control software to be able to observe in high-speed multi-windowing mode.

The sky area available for locating a reference star is limited to $\sim 6.5 \times 6.5 \operatorname{arcmin}^{2}$.

\subsection{Observations and reductions}

Table 4 contains the information related to the time-series observations. We observed the stars in windowed mode using three or more reference stars for constructing the
Table 4. Time-series photometry.

\begin{tabular}{|c|c|c|c|c|}
\hline Object & $\begin{array}{c}\text { Date } \\
(2000)\end{array}$ & Observers & $\begin{array}{l}\text { Start } \\
(\mathrm{UT})\end{array}$ & $\begin{array}{c}\text { Length } \\
(\mathrm{s})\end{array}$ \\
\hline \multirow[t]{3}{*}{ HS $0039+4302$} & Oct. 4 & $\mathrm{R} \varnothing, \mathrm{JES}$ & $23: 07$ & 3880 \\
\hline & Oct. 5 & RØ, JES & $23: 15$ & 7720 \\
\hline & Oct. 7 & $\mathrm{R} \varnothing, \mathrm{JES}$ & 01:35 & 9100 \\
\hline \multirow[t]{3}{*}{ HS $0444+0458$} & Oct. 6 & RØ, JES & $02: 27$ & 3240 \\
\hline & Oct. 7 & RØ, JES & $04: 55$ & 5560 \\
\hline & Oct. 8 & RØ, JES & $02: 37$ & 5260 \\
\hline \multirow[t]{3}{*}{ HS $1824+5745$} & Jul. 9 & $\mathrm{R} \varnothing, \mathrm{RS}$ & $00: 52$ & 4100 \\
\hline & Jul. 10 & $\mathrm{R} \varnothing, \mathrm{RS}$ & $00: 23$ & 3800 \\
\hline & Jul. 11 & $\mathrm{R} \varnothing, \mathrm{RS}$ & $00: 22$ & 2820 \\
\hline \multirow[t]{3}{*}{ HS $2151+0857$} & Jul. 9 & $\mathrm{R} \varnothing, \mathrm{RS}$ & 03:14 & 4360 \\
\hline & Jul. 10 & $\mathrm{R} \varnothing, \mathrm{RS}$ & 02:49 & 5040 \\
\hline & Jul. 11 & $\mathrm{R} \varnothing, \mathrm{RS}$ & $03: 18$ & 8400 \\
\hline
\end{tabular}

relative light curve. All the observations were made with a standard Bessell $B$-band filter (NOT \#74, Bessell 1990), except for the last observation run of HS $0444+0458$, where we experimented with a much wider filter that effectively encompass all bands from $B$ to $R$ with more than $90 \%$ transmission (NOT \#92) ${ }^{1}$. This filter (hereafter referred to as the $W$ filter) has the same center as that of the $V$-band $(5500 \AA)$, but is a full $2750 \AA$ wide. The cycle time was set to $20 \mathrm{~s}$, except for the first run of HS $0444+0458$, where the cycle time was set to $30 \mathrm{~s}$. For the $20 \mathrm{~s}$ cycles, the actual exposure time varied between 15.6 and $16.4 \mathrm{~s}$,

\footnotetext{
1 Filter details with transmission curves for all filters available at the NOT can be found on their web-pages; http://www.not.iac.es/.
} 


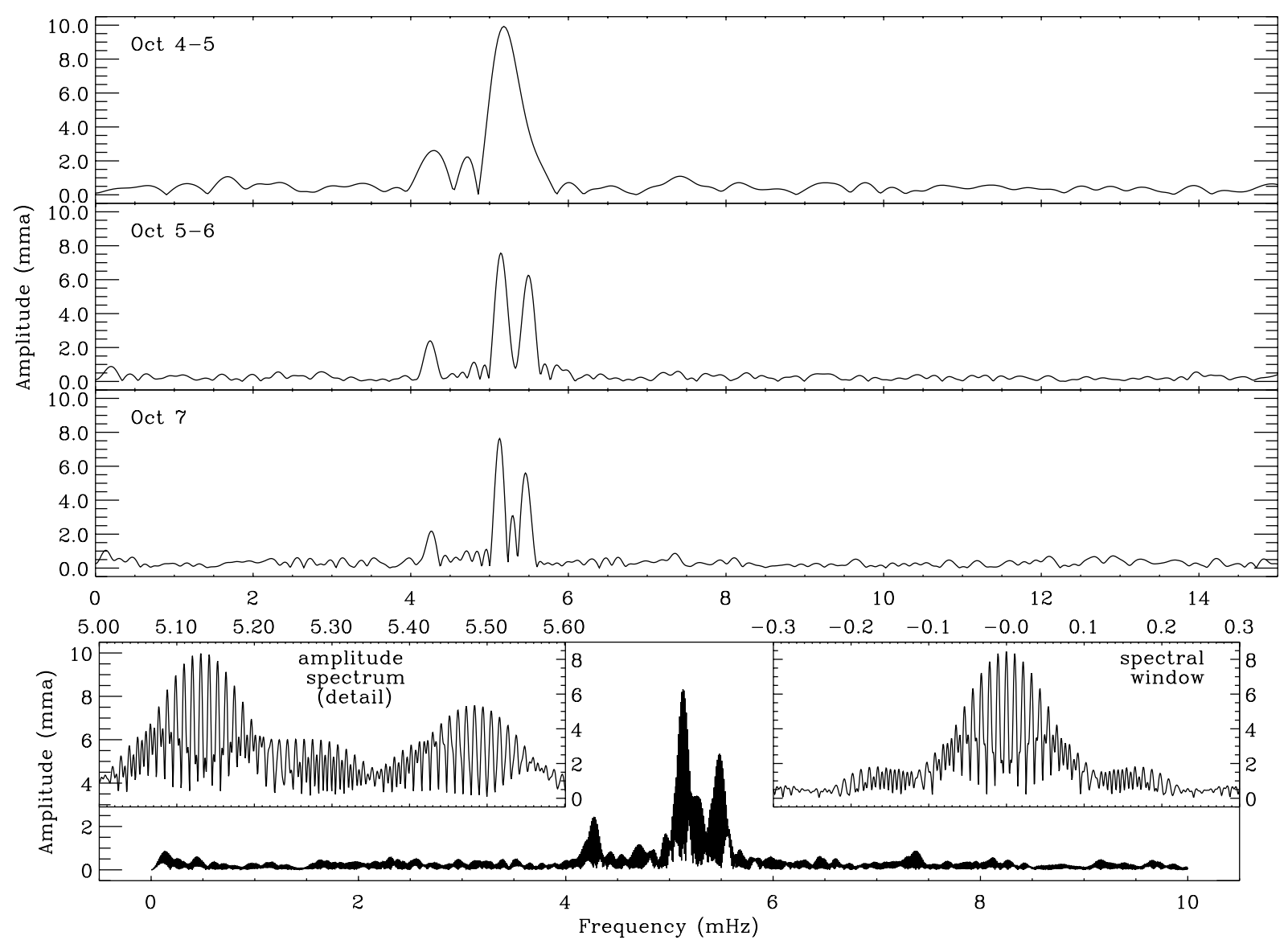

Fig. 4. Amplitude spectra for HS 0039+4302. The three upper panels show the individual spectra for the three nights, and the lower panel shows the spectrum of the three consecutive nights taken together. The two inset panels represent a section of the same spectrum with a larger scale (left) and its spectral window (right).

depending on the number of reference stars selected $(3$ or 4$)$ and the sizes of the readout windows $(42 \times 42$ to $52 \times 52$ pixels).

The data were reduced on-line using the Real Time Photometry (RTP) program developed by one of us (R. Østensen) as part of his Ph.D-project (Østensen 2000). Some details about this software are given in our previous paper (Østensen et al. 2001).

The processing includes bias level removal, flat fielding, sky subtraction, extinction correction and aperture photometry using optimal apertures that track each stars geometrical center. The resulting light curves are shown in Figs. 3, 5, 7 and 9, and are discussed below for each of the four stars. The light curves show differential photometry between the target and the best combination of the available reference stars.

The optimal aperture for each run was selected after processing all data sets with apertures of a wide range of diameters and choosing the one that gave the best signalto-noise ratio in the Fourier Transform (FT), using the amplitude of the primary peak for the signal and the mean of the amplitude spectrum outside the pulsation range for the noise. The apertures tested ranged between 10 and 40 pixels, which corresponds to 1.9 and 7.6 arcsec on the sky, while the best results stayed within the range 18 to 22 pixels (3.4 to 4.2 arcsec).
Figures 4, 6, 8 and 10 show the FTs of the light curves. The significant peaks detected in these amplitude spectra are listed in Table 3.

\subsection{Data analysis and results}

\subsubsection{HS 0039+4302}

Since the runs from the three nights have progressively longer time spans, the corresponding amplitude spectra reveal increasing detail. The dominant period is seen at $5.14 \mathrm{mHz}$, with an amplitude of about $8 \mathrm{mma}$. In the amplitude spectrum of the third night, four significant peaks are revealed (see Table 3 for details). In the bottom panel of Fig. 4, the combined FT of all the three nights taken together is shown. This spectrum agrees with what is seen in the third night alone, but reveals no further significant peaks.

Figure 3 shows the observed data superimposed on a synthetic light curve computed from the four detected pulsation periods. The match between these are quite good, although the amplitude spectra show some remaining power close to the observed oscillation frequencies (Fig. 4). The peak at $4.6 \mathrm{mHz}$ in the amplitude spectrum of the first run vanishes completely after prewhitening. 


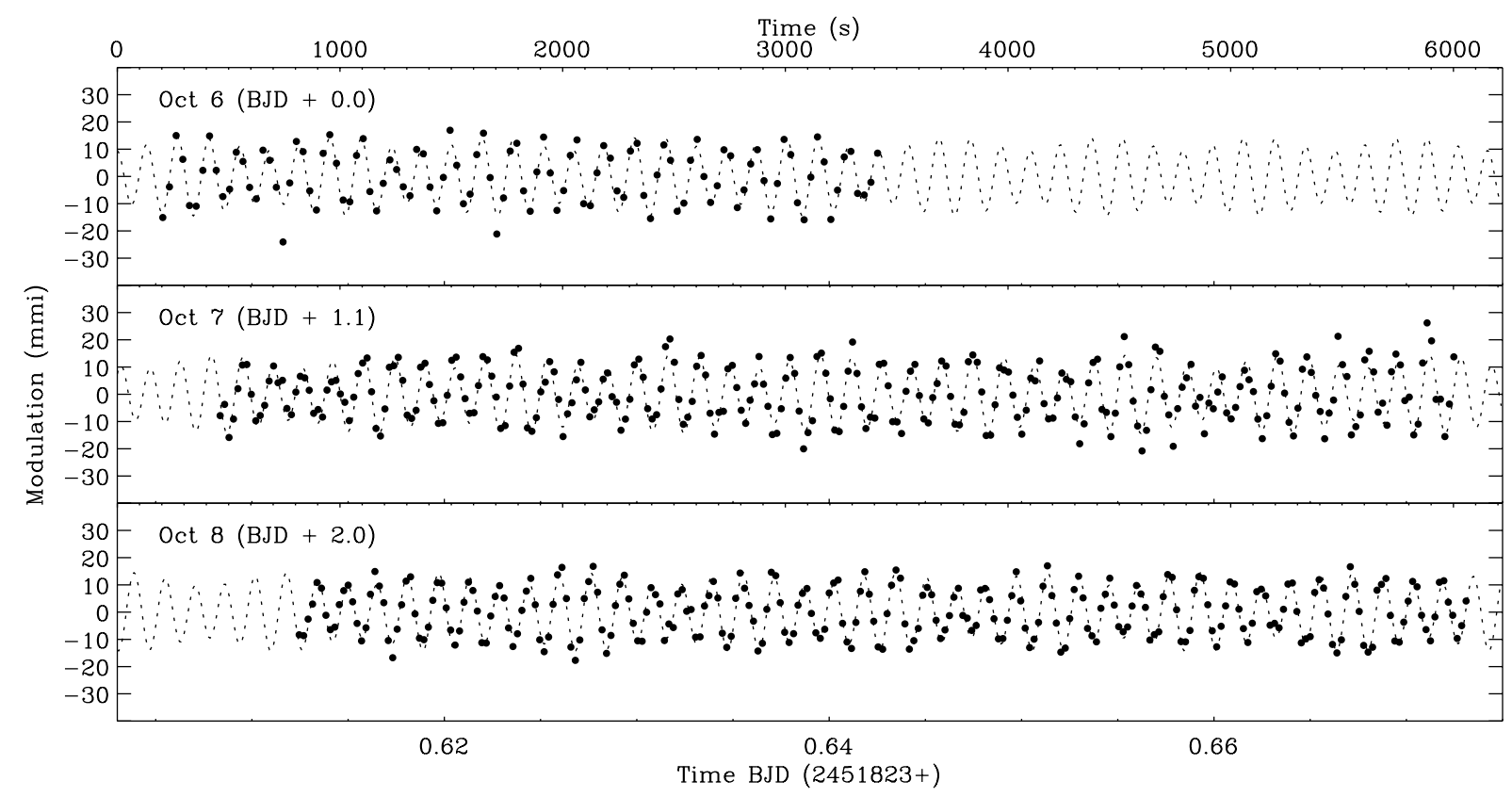

Fig. 5. Observed and synthetic light curves for HS 0444+0408. Note that since the observations on the second night were done 2 hours later in the night, the panel has been shifted by 0.1 JD relative to the bottom scale.

The average noise level in the amplitude spectra after prewhitening of the four detected periods is below $0.5 \mathrm{mma}$ for the first run, below $0.3 \mathrm{mma}$ for the second, and about 0.35 in the third run. The average noise level in the combined spectrum is below 0.25 mma.

\subsubsection{HS $0444+0408$}

In the first run on HS 0444+0408, a sequence interval of $30 \mathrm{~s}$ was used. After the discovery of the clear pulsation on the first night, we decreased the sampling interval to $20 \mathrm{~s}$ for the second and third runs, to better sample the light curve. On the third night we observed this target using the $W$-band filter, in an attempt to improve the signal-tonoise ratio. In this way the white noise in the FT dropped from $0.5 \mathrm{mma}$ in the second run to 0.35 in the third, even when the run length in the third run was slightly shorter than in the second.

Both the significant peaks detected, the dominating one at $7.31 \mathrm{mHz}$ and the secondary one at $5.86 \mathrm{mHz}$, have a quite stable amplitude over the three nights of 11-12 and 2.3-2.5 mma respectively. In particular, the amplitudes of both peaks in the third night, when we used the $W$ filter, are almost identical to the amplitudes obtained in the first two nights, when the $B$ filter was used. Therefore we could do a combined FT using the data from all the three nights together.

The synthetic light curve based on the two detected periods, and plotted with a dotted line in Fig. 5, reproduces the observed light curve very well.

In the bottom panel of Fig. 6, the combined FT of the three nights is shown. The feature between the two main periods is just above the detection limit, at $1.4 \mathrm{mma}$.
The average noise level in the combined spectrum is below $0.3 \mathrm{mma}$.

The extinction corrected light curves indicate an average magnitude $B=15.4$ for both nights with $B$-band data, in good agreement with the estimates based on the photographic plates.

\subsubsection{HS $1824+5745$}

This target and the following show pulsation amplitudes of less than half of the previous two. For this reason the light curves and amplitude spectra have been scaled to show as much detail as possible.

The Fourier transforms of HS $1824+5745$ show no indications of more than one single period at $7.21 \mathrm{mHz}$. However, the synthetic light curve produced from the detected period does not reproduce the observed light curve as well as what was found for the two previous sdB pulsators (see Fig. 7). The noise in the data is higher in these observations, due to a combination of poorer weather conditions and a fainter target. Note that since the pulsations are much weaker, the $y$-axis scale in Fig. 7 has been stretched relative to the light curves in Figs. 3 and 5. Note also that the last third of the first night contains data of poorer quality than the first part, due to an onset of clouds that eventually forced the termination of observations. The second night has some slight cirrus activity throughout the run, while the third night is photometric.

The amplitude appears to be variable, changing from 2.6 to $4.8 \mathrm{mma}$ from the first to the third night, but this is uncertain due to the poor conditions on the first two nights and the short length of the runs. For instance, the amplitude for the first night can be brought in accordance 


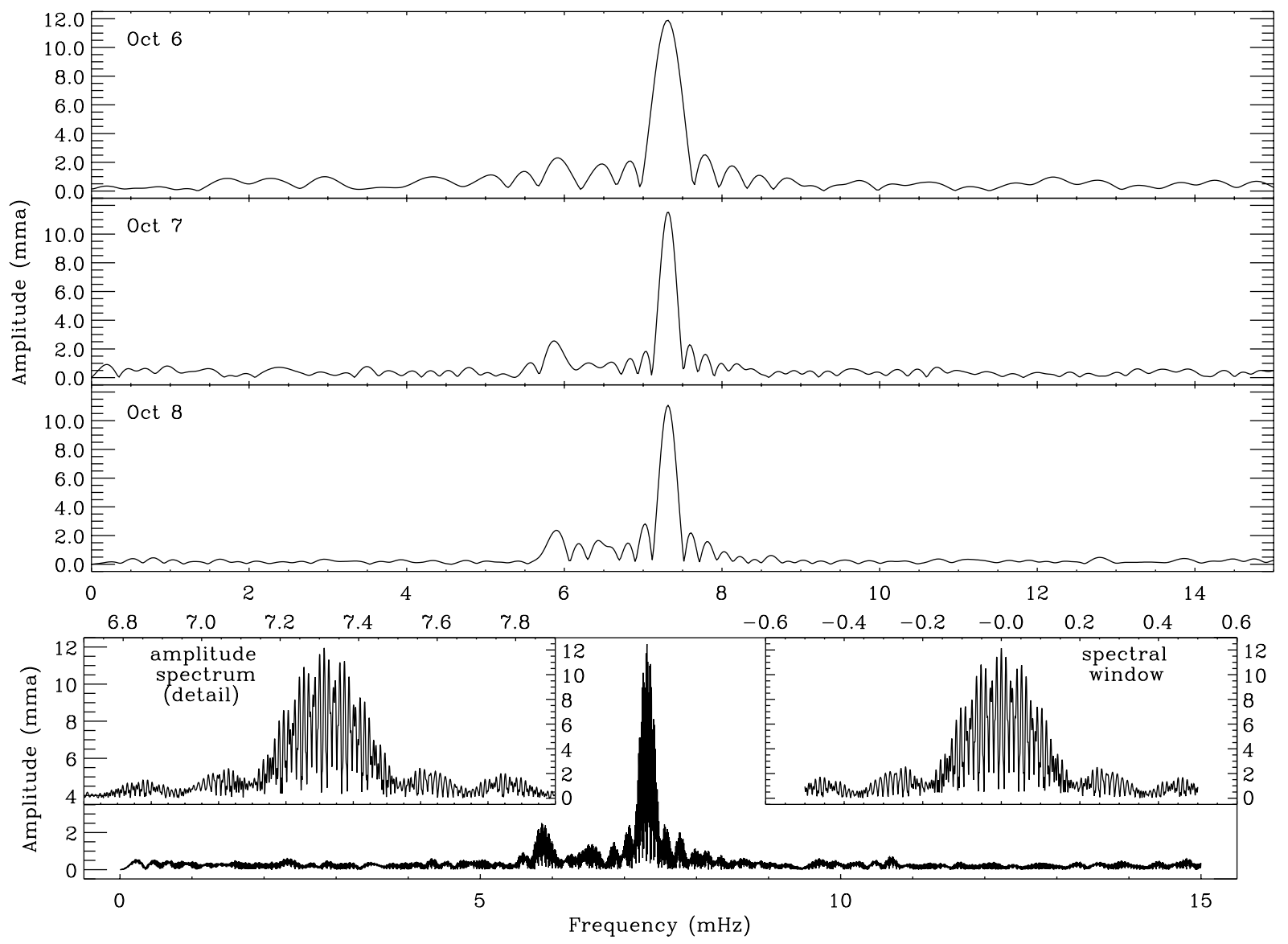

Fig. 6. Amplitude spectra for HS $0444+0408$. The three upper panels show the individual spectra for the three nights, and the lower panel shows the spectrum of the three consecutive nights taken together. The two inset panels represent the same spectrum with a larger scale (left) and its spectral window (right).

with that of the whole run by taking the FT of only the first (good) two thirds of the sequence.

\subsubsection{HS $2151+0857$}

This object shows a richer period spectrum than HS $1824+5745$, and clearly reveals four periods at 6.59 , 6.86, 7.43 and $7.72 \mathrm{mHz}$ (see Fig. 10), with amplitudes between 2 and 5 mma, as listed in Table 3 .

After prewhitening of the four tabulated frequencies, the only significant feature in the combined amplitude spectrum is a $1.4 \mathrm{mma}$ peak at $7.72 \mathrm{mHz}$, only $6 \mu \mathrm{Hz}$ away from the $3.3 \mathrm{mma}$ peak. This feature may not be real, since the amplitude of this peak in the individual spectra appears to change considerably from the first to the third night. The synthetic light curve trace the obseved data reasonably well, but some discrepancies are seen. Some of this is due to noise, since this target is about one magnitude fainter than the three others, but it is also likely that unresolved periods or changes in period amplitudes over the three nights are significant.

The mean noise level in the prewhitened spectra is below $0.8 \mathrm{mma}$ in the first night, below $0.7 \mathrm{mma}$ in the second, and below $0.6 \mathrm{mma}$ in the third. In the prewhitened spectrum of all three nights together the mean is below 0.4 .
The prescision of the observations decreases somewhat in the last quarter of the third run, due to the onset of twilight.

\section{Summary and discussion}

The four new pulsating sdB stars presented here are all located well inside the region of the $\left(T_{\text {eff }}, \log g\right)$ plane where the sdB pulsators are expected, and have fairly simple temporal spectra, as far as we can judge from our short light curves.

HS $0039+4302$, with a $T_{\text {eff }}$ of 32400 and $\log g$ of 5.7 , is located right in the centre of the $\operatorname{sdBV}\left(T_{\text {eff }}, \log g\right)$ distribution, comparable to PG 1336-018 (Kilkenny et al. 1998). The main peak of PG 1336-018 is found at $184 \mathrm{~s}$, with a secondary at $141 \mathrm{~s}$. For HS $0039+4302$ most of the power appears to be in a group of peaks between 195 and $182 \mathrm{~s}$, and a secondary group is seen at $242 \mathrm{~s}$. The rich pulsation spectrum and relatively strong amplitudes of this target makes it our favourite target for a multi-site campaign. HS $0039+4302$ is also the brightest among these new pulsators $(B=15.1)$.

HS 0444+0408, with $T_{\text {eff }} \simeq 33800 \mathrm{~K}$ and $\log g \simeq$ 5.6 , is similar to KPD $1930+2752$ (Billères et al. 2000). Campaign observations of KPD $1930+2752$ have revealed that its pulsation spectrum contains at least 44 periods 


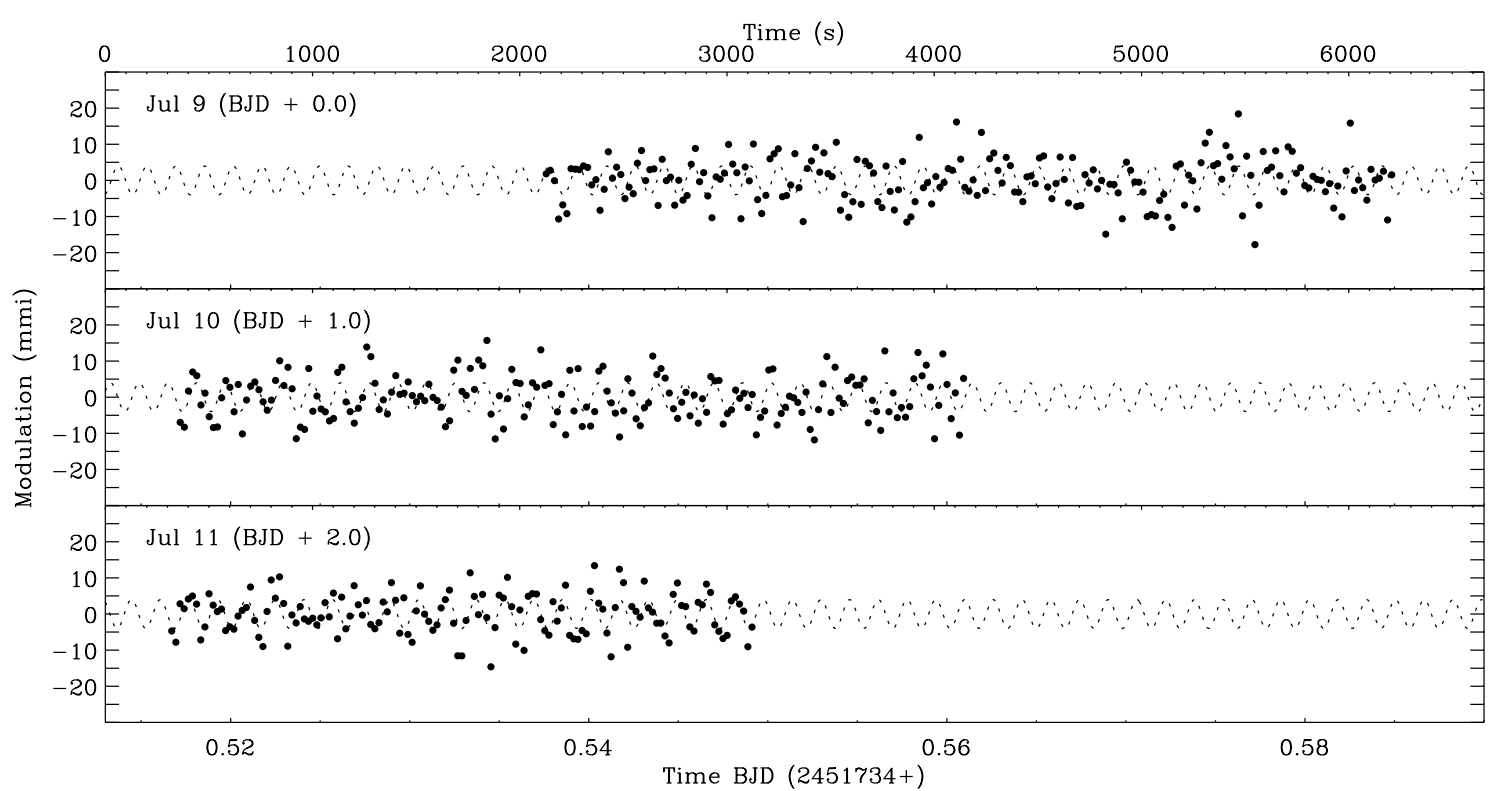

Fig. 7. Observed and synthetic light curves for HS $1824+5745$.

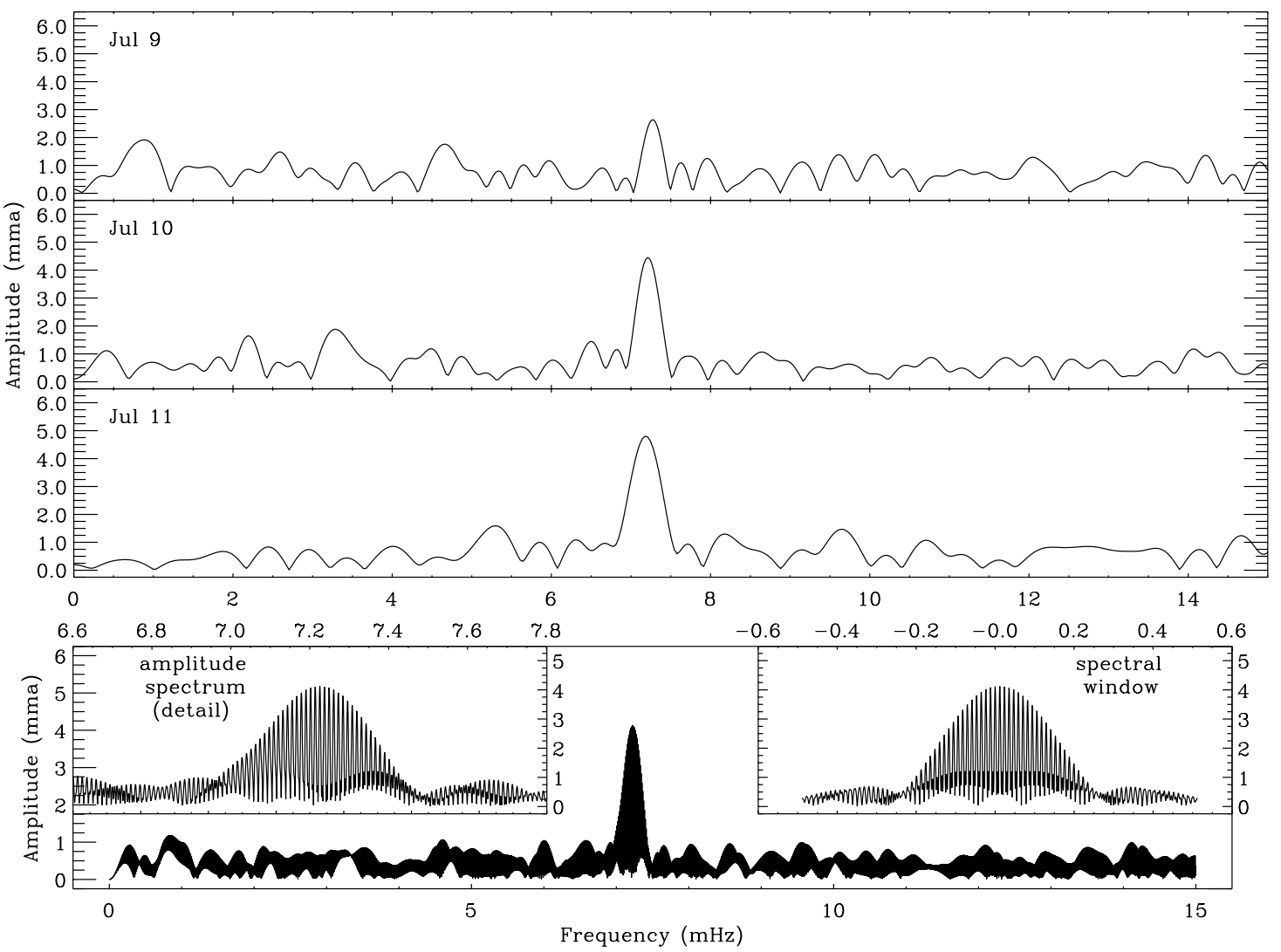

Fig. 8. Amplitude spectra for HS $1824+5745$. The three upper panels show the individual spectra for the three nights, and the lower panel shows the spectrum of the three consecutive nights taken together. The two inset panels represent the same spectrum with a larger scale (left) and its spectral window (right).

located in a number of discrete groups between 145 and 332 s (Billères et al. 2000). Our three short observing runs on HS $0444+0408$ reveal one clear pulsation around $137 \mathrm{~s}$, and at least one more close to $170 \mathrm{~s}$. Higher frequency resolution observations of this star could reveal if further periodicities are present.
HS $1824+5745$ has $T_{\text {eff }} \simeq 33100 \mathrm{~K}$ and $\log g \simeq 6.0$, which places it in the mid temperature - high gravity part of the $\operatorname{sdBV}\left(T_{\text {eff }}, \log g\right)$ distribution, right next to EC 10228-0905 (Stobie et al. 1997). Whereas we only detect a single peak at $139 \mathrm{~s}$ in the amplitude spectrum of HS 1824+5745, EC 10228-0905 displays three peaks in the 


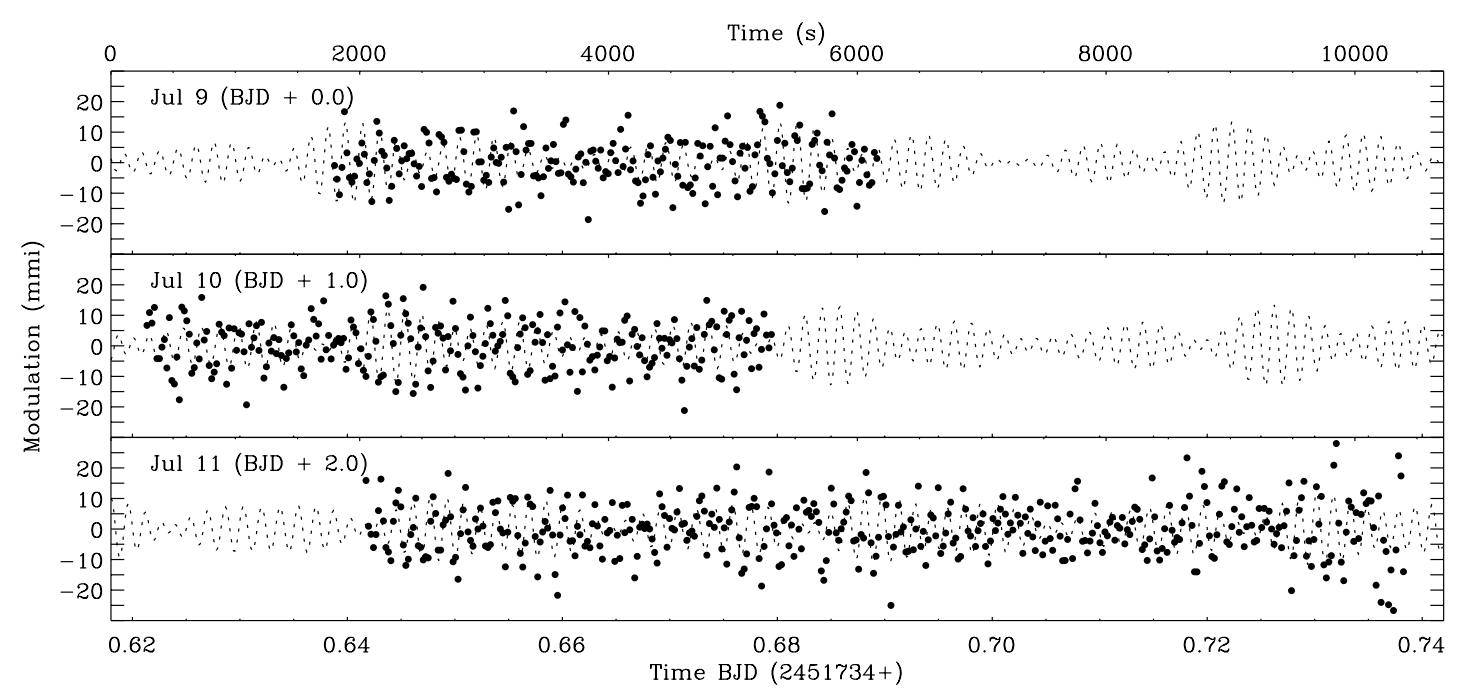

Fig. 9. Observed and synthetic light curves for HS $2151+0857$.

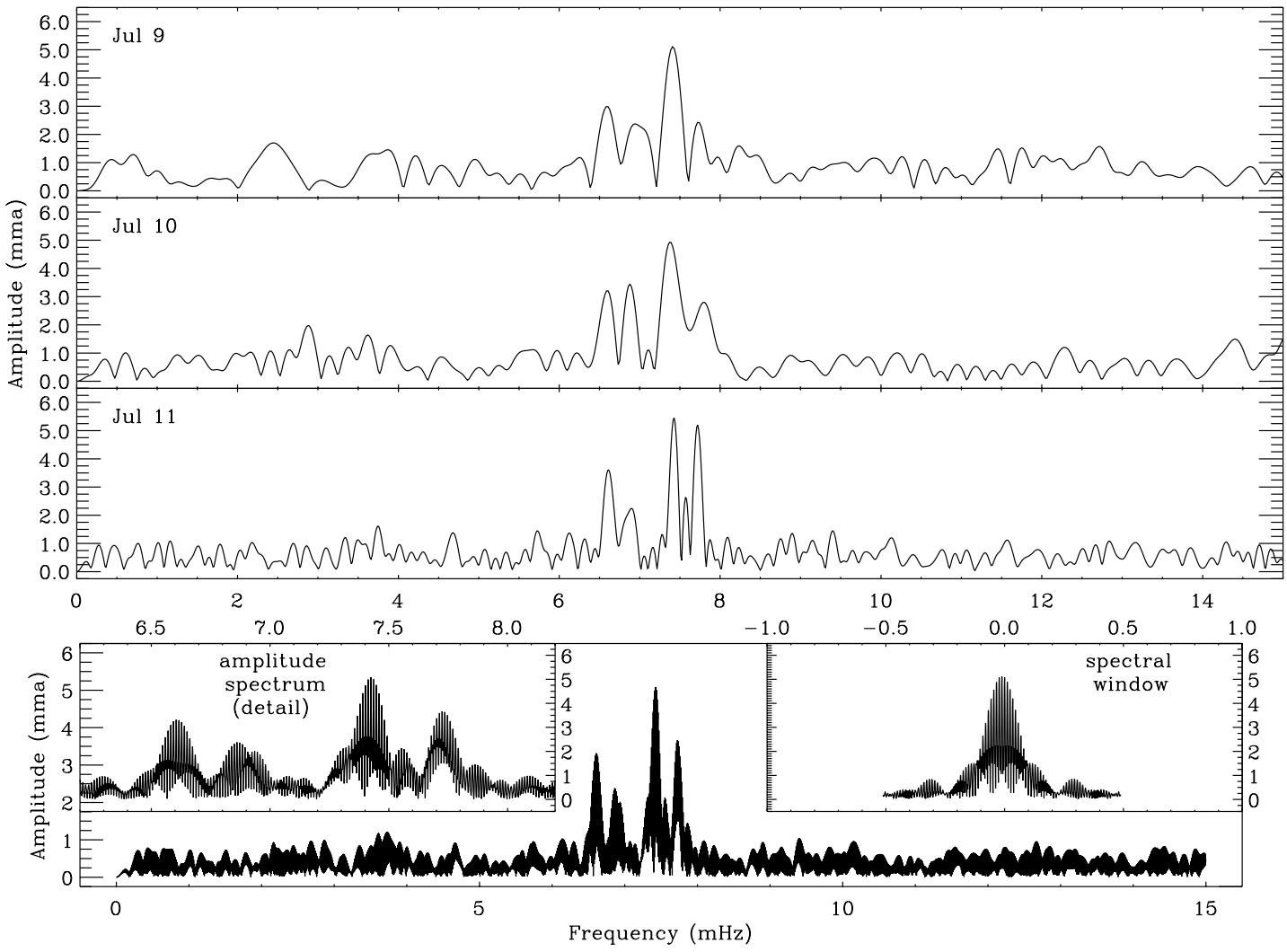

Fig. 10. Individual and combined amplitude spectra for HS $2151+0857$.

range $139-152 \mathrm{~s}$ with the most significant peak at $139 \mathrm{~s}$. Also, the observations reported by Stobie et al. (1997) indicate that the amplitudes are variable, just as we can see for HS $1824+5745$. Thus, a comparison between these two stars indicates a link between $\left(T_{\text {eff }}, \log g\right)$ and pulsational behaviour.

HS $2151+0857$ has almost identical $T_{\text {eff }}, \log g$ and pulsation periods as the class prototype EC 14026-2647, which has at least two periods where one is stable and the other apparently variable in amplitude (Kilkenny et al. 1997). In the three nights we observed HS 2151 the main peak remained stable, while at least one peak (at $7.72 \mathrm{mHz}$ ) varied from about 2.6 to $4.6 \mathrm{mma}$, giving at least some indication of corresponding amplitude variability to that of the prototype. The periods detected for HS 2151+0857 (between 129 and 151s) also span nearly the same range as that of EC 14026-2647 (134 and 144s).

The new pulsators all follow the $\log g-P$ relation (see Koen et al. 1999), as we see from Fig. 11, where $\log g$ has been plotted against the period of the pulsation with the strongest amplitude.

Acknowledgements. This research was supported by the Norwegian Research Council. R. S. acknowledges support by 


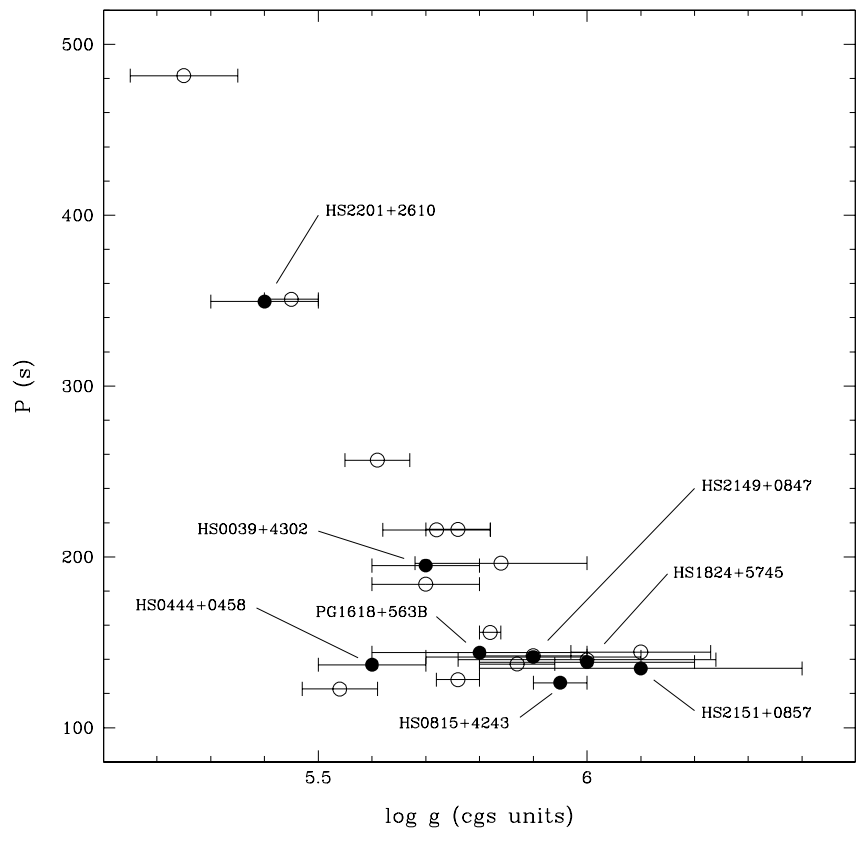

Fig. 11. In this figure $\log g$ values are plotted versus the pulsation period with largest detected amplitude. The eight pulsators found during our NOT search-programme are represented with filled circles.

the italian research foundation MURST under project "Stellar Evolution" (coordinator V. Castellani). H. E. acknowledges financial support by the german research foundation DFG under grant He 1354/30-1. The authors also wishes to thank the staff at the NOT for good support. S. D. and U. H. thank D. Engels, H. Hagen and D. Reimers (Hamburg) for a fruitful collaboration over many years.

The time-series data presented here have been taken using ALFOSC, which is owned by the Instituto de Astrofisica de Andalucia (IAA) and operated at the Nordic Optical Telescope under agreement between IAA and the NBIfAFG of the Astronomical Observatory of Copenhagen.

This research has made use of the SIMBAD database, operated at CDS, Strasbourg, France.

\section{References}

Abrahamian, H. V., Lipovetsky, V. A., Mkaelian, A. M., \& Stephanian, J. A. 1990, Afz, 33, 345

Bessell, M. S. 1990, PASP, 102, 1181

Billères, M., Fontaine, G., Brassard, P., et al. 2000, ApJ, 530, 441

Bixler, J. V., Bowyer, S., \& Laget, M. 1991, A\&A, 250, 370

Brown, T. M., Ferguson, H. C., Davidsen, A. F., \& Dorman, B. 1997, ApJ, 482, 685

Brown, T. M., Bowers, C. W., Kimble, R. A., Sweigart, A. V., \& Ferguson, H. C. 2000, ApJ, 532, 308

Charpinet, S., Fontaine, G., Brassard, P., \& Dorman, B. 1996, ApJ, 471, L106

Charpinet, S., Fontaine, G., Brassard, P., et al. 1997, ApJ, 483, L123

Dorman, B., O’Connell, R. W., \& Rood, R. T. 1993, ApJ, 419, 596

Edelmann, H., Heber, U., Lemke, M., et al. 2001, A\&A, in preparation
Ferraro, F. R., Paltrinieri, B., Fusi Pecci, F., et al. 1997, ApJ, 484, L145

Fontaine, G., Charpinet, S., Brassard, P., et al. 1998, New Eyes to See Inside the Sun and Stars, IAUS, 185, 367

Green, R. F., Schmidt, M., \& Liebert, J. 1986, ApJS, 61, 305

Greggio, L., \& Renzini, A. 1999, Mem. S. A. It. [astro-ph/9811131]

Hagen, H-J., Groote, D., Engels, D., \& Reimers, D. 1995, A\&AS, 111, 195

Heber, U. 1986, A\&A, 155, 33

Heber, U., Edelmann, H., Lemke, M., Napiwotzki, R., \& Engels, D. 1999, PASPC, 169, 551

Heber, U., Reid, I. N., \& Werner, K. 2000, A\&A, 363, 198

Kawaler, S. D. 1999, in Proc. of the 11th European Workshop on White Dwarfs, ed. J.-E. Solheim, \& E. G. Meištas, ASP Conf. Ser., 169, 158

Kilkenny, D., Heber, U., \& Drilling, J. S. 1988, South African Astron. Obs. Circ., 12, 1

Kilkenny, D., Koen, C., O’Donoghue, D., \& Stobie, R. S. 1997, MNRAS, 285, 640

Kilkenny, D., O’Donoghue, D., Koen, C., Lynas-Gray, A. E., \& Van Wyk, F. 1998, MNRAS, 296, 329

Kilkenny, D., Koenn, C., O'Donoghuen, D., et al. 1999, MNRAS, 303, 525

Koen, C., O'Donoghue, D., Kilkenny, D., et al. 1998, MNRAS, 296, 317

Koen, C., Kilkenny, D., O'Donoghue, D., Van Wyk, F., \& Stobie, R. S. 1997, MNRAS, 285, 645

Koen, C., O'Donoghue, D., Pollacco, D. L., \& Nitta, A. 1998, MNRAS, 300, 1105

Koen, C., O'Donoghue, D., Pollacco, D. L., \& Charpinet, S. 1999, MNRAS, 305, 28

Lemke, M., Heber, U., Napiwotzki, R., Dreizler, S., \& Engels, D. 1997, in The third conference on Faint Blue Stars, ed. A. G. D. Philip, J. W. Liebert, \& R. A. Saffer (L. Davis press), 375

Maxted, P. F. L., Heber, U., Marsh, T. R., \& North, R. C. 2001, MNRAS, in press [astro-ph 0103342]

Napiwotzki, R. 1997, A\&A, 322, 256

O'Donoghue, D., Koen, C., \& Solheim, J.-E. 1998, MNRAS, 296, 296

O'Donoghue, D., Koen, C., Lynas-Gray, A. E., Kilkenny, D., \& Van Wyk, F. 1998, MNRAS, 296, 306

O'Donoghue, D., Koen, C., Kilkenny, D., \& Stobie, R. S. 1999, in Proc. of the 11th European Workshop on White Dwarfs, ed. J.-E. Solheim, \& E. G. Meištas, ASP Conf. Ser., 169, 149

Østensen, R., \& Solheim, J.-E. 2000, in ed. G. Vauclair, \& E. G. Meištas, Proc. of the 5th Whole Earth Telescope Workshop, Baltic Astron., 9, 411

Østensen, R. 2000, Time Resolved CCD Photometry, Ph.D. Thesis, University of Troms $\varnothing$

Østensen, R., Solheim, J.-E., Heber, U., et al. 2001, A\&A, 368, 175

Piccioni, A., Bartolini, C., Bernabel, S., et al. 2000, A\&A, 354, L13

Silvotti, R., Solheim, J.-E., Gonzalez-Perez, J. M., et al. 2000a, A\&A, 359, 1068

Stobie, S., Kawaler, S. D., Kilkenny, D., O'Donoghue, D., \& Koen, C. 1997, MNRAS, 285, 651

Ulla, A., Zapatero Osorio, M. R., Pérez Hernández, \& MacDonald, J. 2001, A\&A, in press [astro-ph/0102142] 